\title{
Is iron deficiency a risk factor for postpartum depression? A case-control study in the Gaza Strip, Palestine
}

\author{
Samar Hameed ${ }^{1, *}$, Ihab A Naser ${ }^{1,2}{ }^{\infty}$, Mohamed A Al Ghussein $^{1}$ and \\ Mohammed S Ellulu 1,2 \\ 'Master Program in Clinical Nutrition, Faculty of Pharmacy, Al-Azhar University, Gaza, Palestine: ${ }^{2}$ Clinical Nutrition \\ Department, Faculty of Applied Medical Sciences, Al-Azhar University, Gaza, Palestine
}

Submitted 20 0ctober 2020: Final revision received 12 August 2021: Accepted 26 August 2021: First published online 31 August 2021

\begin{abstract}
Objective: This study aims to investigate the association between iron body status and postpartum depression (PPD) among mothers during the postpartum period. Design: This is a case-control study.

Setting: Governmental primary health care centres in the Gaza Strip, Palestine.

Participants: This study involved 300 mothers a month after delivery, with one 150 mothers that were recruited in the cases group who were diagnosed with PPD based on Edinburgh Postnatal Depression Scale (EPDS) $\geq 10$. The control group included 150 mothers did not have PPD (EPDS < 10). Body iron status is represented by the index of sTfR/log ferritin.

Results: Among PPD mothers, $43.3 \%$ had low ferritin level $v .15 \cdot 3 \%$ for controls $(P<0.001)$ and cases $v$. controls difference in mean Hb level was $-0.61(95 \%$ $\mathrm{CI}-0 \cdot 86,-0 \cdot 35)$. The results of the multiple logistic regression reported that there is a statistically significant association between PPD and the body iron status existed, as mothers who suffered from iron deficiency (ID) were three times more likely to have PPD (OR $\left.{ }^{\text {adj }} 3.25 ; P=0.015\right)$. Furthermore, the results of the final regression model showed that the other factors that can lead to PPD are absence of psychological guidance services (OR $\left.{ }^{\text {adj }} 8.54 ; P=0.001\right)$, suffering from undesired feeling in the last pregnancy $\left(\mathrm{OR}^{\text {adj }} 1.77 ; P=0.034\right)$, in addition to having one of the mental health disorders in the last pregnancy $(P=0.001)$.

Conclusion: Body iron status might be a risk factor for postpartum depression and other possibilities of reverse causality may worsen the condition.
\end{abstract}

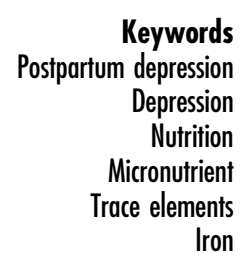

The WHO considered iron deficiency (ID) as one of the most common forms of nutritional deficiencies, especially among females affecting $33 \%$ of non-pregnant women, $40 \%$ of pregnant women and $42 \%$ of children worldwide $^{(1)}$. ID during pregnancy can cause widespread and significant consequences for both the mother and her infant's physiological and mental health ${ }^{(2)}$.

Iron has several vital functions in the body including the functions of the iron-containing enzymes, the synthesis of bile acid and steroid hormones, and signal -controlling in some neurotransmitters, such as the serotonin and dopamine systems in brain ${ }^{(3)}$. ID has adverse effects on areas of women's mental health such as mood, short-term memory, verbal learning, attention, concentration and intelligence. It can also lead to depression ${ }^{(4)}$.

Various physiological variables related to ID that contribute to postpartum depression (PPD) such as fatigue that

have been considered as significant predictor of $\mathrm{PPD}^{(5)}$. The next variable is anaemia which contributes to fatigue and is associated with additional symptoms such as irritability, apathy and an inability to concentrate ${ }^{(6)}$. Furthermore, ID has been found to have a role in altering thyroid hormone metabolism ${ }^{(7)}$. All of these symptoms (fatigue, anaemia and abnormal thyroid status) are probably interrelated in certain individuals; if they occur during the postpartum period, they could affect maternal psychological and health outcome.

PPD is a major public health concern that may lead to the most common complications of childbearing and represents a serious health problem affecting mothers and their families, and thus the whole society, as it affects about $10-15 \%$ of new mothers ${ }^{(8)}$. However, depression in women during the postpartum period may start during pregnancy or may have onset beyond the first postpartum 
month $^{(9)}$. The exact aetiology of PPD is still unclear; however, it is generally thought to be linked to genetic, biological, hormonal, psychosocial, environmental and nutritional factors $^{(10)}$.

Generally, balanced nutrition plays an important role in the model of thinking and behaviour, and affects the cognition and memory capacity, and has direct or indirect effects on brain health; as the intake of different foods is directly involved in the synthesis and metabolism of related neurotransmitters ${ }^{(11)}$.

During pregnancy and postpartum periods, mothers face continuous nutritional vulnerability, since malabsorption, food choice, food preparation and other socioeconomic factors collectively may have a reverse effect on the micronutrients level including iron ${ }^{(12)}$. Moreover, due to the physiological changes during pregnancy, ID often occurs towards the end of pregnancy even among women who enter pregnancy with some iron stores, which extends to the postpartum period that is associated with the development of maternal PPD ${ }^{(13)}$.

Generally, there are discrepancies in the literature concerning the association between iron and PPD. The direct relationship between ID biomarkers and PPD has been confirmed in different studies ${ }^{(14-16)}$. This relation stems from the fact that iron is critical for adequate myelination, neurotransmitter metabolism and function, and neuronal cellular and oxidative processes ${ }^{(17)}$. In contrast, a study established in China found that there is no relationship between maternal iron status and PPD ${ }^{(18)}$.

This study was conducted in Gaza City, Palestine, due to some perturbing factors. Gaza had been insecure since 2009, due to the dramatic series of events in the region ${ }^{(19)}$. In addition to the socio-economic situation and food insecurity, the Palestinians are exposed to political violence that makes them at heightened risk for major depression and post-traumatic stress disorder. This makes depression one of the top five causes of disability in the occupied Palestinian territory ${ }^{(20)}$. It leads to low socio-economic status resulting in micronutrients deficiencies. This study aims to investigate the association between iron body status and PPD among mothers during the postpartum period in Gaza city.

\section{Methods}

\section{Study design and sampling}

The study population was obtained from the immunisation electronic records of the newly registered neonates in postnatal care. The study involved postpartum mothers, a month (30 d) after delivery, who visited the randomly selected (five out of ten) governmental primary health care centres with their infants for Polio vaccines.

A case-control study was conducted in the period from January 2020 to August 2020 in which 1100 postpartum mothers were screened for PPD by using a validated
Edinburgh Postnatal Depression Scale (EPDS). The cases were postpartum mothers with depression who scored EPDS $(\geq 10)$, while mothers who scored EPDS $(<10)$ were not diagnosed with PPD disorder and considered as controls. The controls were paired with the cases according to age and place of residency. Participants who were taking psychiatric drugs during the pregnancy period were excluded from the study. The additional exclusion criteria included mothers who had a history of postpartum blood transfusion, had a postpartum hemorrhage, smokers - as smoking may alter the $\mathrm{Hb}$ ratio and increase the oxidative stress - and mothers who refused to sign the consent form.

The study involved 300 mothers, as the sample size was calculated using Power Statistics program for sample size calculation (PS) and by using two-proportion method in Power Statistics software. P0 is the possibility of exposure in the control group $=23.3 \%$ based on the prevalence of ID among non-PPD mothers according to Albacar and his colleagues, while $\mathrm{P} 1=38.8 \%$ indicating that 38.8 of the PPD had ID ${ }^{(14)}$. The calculated sample size according to PS software was 144 in each group. After considering the nonresponse rate, the number of the subjects in each group was increased to 150 .

\section{Questionnaire}

A face-to-face interview was conducted to complete a questionnaire designed to meet the needs of the case and control groups. During the interview, the researcher clarified questions for participants. Most questions were closed-ended questions. The questionnaire is divided into three parts: the first part deals with socio-demographic and socio-economic characteristics, the second part contains obstetric and gynecological history, the third part includes the biochemical section.

\section{Assessment of PPD}

The translated copy of EPDS used in this study was established by the Department of Health Government of Western Australia. The cut-off point, recommended by the author, was $9 / 10$, which means a score of 10 or higher indicates that depressive symptoms have been reported. The validation study of this translated questionnaire on Arabic mothers showed that the sensitivity and specificity for the selected cut-off point $9 / 10$ were $91 \%$ and $84 \%$, respectively ${ }^{(21)}$. Thus, the mothers who scored EPDS $(\geq 10)$ were diagnosed with PPD disorder, while mothers who scored EPDS $(<10)$ were not diagnosed with PPD disorder.

\section{Blood samples and biochemical measurements}

The blood sample collection from the mothers occurred at the newborn's vaccination section in the selected clinics. The samples were drawn carefully to avoid haemolysis. A 5-ml venous blood sample was drawn from each participant. About 1 and a $1 / 2 \mathrm{ml}$ were discharged in EDTA 
vacutainer tubes and mixed gently for the complete blood count test (CBC). The rest of the blood was discharged into the vacutainer plain tube for the biochemical tests and was left to clot then centrifuged at a speed of $3000 \mathrm{rpm}$ for 10 min under room temperature. Then the serum was placed in plain tubes, and in order to avoid loss of bioactivity and contamination, the tubes were sealed and stored at $-20^{\circ} \mathrm{C}$ until the test performed. The biochemical tests that were done from the serum sample are ferritin, hs-CRP and soluble transferrin receptors (sTfR). Each device was calibrated, and controls were performed before each run of samples. All the blood tests were conducted at the Palestinian Medical Relief Society (PMRS) laboratory by the researcher herself.

\section{Assessment of body iron}

In this study, instead of performing the serum iron test, body iron stores were calculated. According to the report of a joint WHO and CDC on the assessment of iron status at the population level, the following equation was used to represent the body iron stores ${ }^{(22)}$ :

$$
\frac{-[\log 10(\text { sTfR } \times 1000 / \text { ferritin })-2.8229]}{0.1207}
$$

According to Cogswell and her colleagues, after applying the previous equation, the estimate of the prevalence of ID defined as body iron $<0 \mathrm{mg} / \mathrm{kg}$; moreover, they compared these estimates with ID based on the ferritin model among females aged between 12 and 49 years. This means that body iron is expressed as iron excess in stores if the result of $\mathrm{sTfR} / \log$ ferritin index was $\geq 0 \mathrm{mg} / \mathrm{kg}$. However, if the result was $<0 \mathrm{mg} / \mathrm{kg}$, then it indicates iron deficit in the body ${ }^{(23)}$

Assessment of serum transferrin receptor levels has been used to distinguish ID anaemia from anaemia of chronic disease because the receptors are generally unaffected by concurrent infection or inflammation ${ }^{(24)}$. Combining the use of serum transferrin receptor concentrations with serum ferritin concentrations as the serum transferrin receptor/log ferritin ratio has also been proposed to increase the diagnostic sensitivity and specificity for diagnosing ID $^{(25,26)}$.

\section{Statistical analysis}

Following data collection, the questionnaires and anthropometric data were reviewed before being entered into the study database. Keyed data were checked for missing or unclear responses following completion of the interview. Respondents were contacted by phone to inquire about unclear and missing data.

Categorical variables like the socio-demographic and obstetric variables were presented as percentages and frequencies, while the quantitative continuous variables, like the biochemical results, were presented as means and standard deviations. The $\chi^{2}$ test was used to compare the distribution of categorical qualitative variables across the PPD and non-PPD groups. Independent $t$-test and Mann-Whitney test were used to compare between means and ranks, respectively. Multiple logistic regression was performed to investigate the association between body iron status and PPD in present of other confounding factors as shown in Fig. 1. The level of significance was set at 0.05.

\section{Results}

The 300 mothers who participated in this study were categorised into 2 groups: the case group that included 150 mothers who were diagnosed with PPD by the EPDS $(\geq$ 10) with a mean score of 15.2 and SD $( \pm 4.2)$ and the control group included 150 mothers who were not diagnosed with PPD by the EPDS $(<10)$ with mean score of 5.26 and SD $( \pm 2 \cdot 65)$.

Table 1 shows that $85 \%$ of cases and $89.3 \%$ of controls were homeowners and the rest of both groups home renters, thus the type of housing $(P=0.856)$ was not significantly different. Neither the educational level nor the occupational status of the mother showed any significant different as $P$-values were 1.00 and 0.289 , respectively. Regarding the total family monthly income for the participant mothers, there was no significant difference between cases and controls $(P=0 \cdot 388)$. The results showed no statistically significant associations between the PPD and the sex of the child $(P=0.298)$. There were no significant differences in proportions between the two groups regarding the worries about the embryo gender $(P=1 \cdot 000)$, having the first baby $(P=0 \cdot 250)$ and planning for the pregnancy $(P=0.468)$. As for the type of delivery, most of the cases and controls had a normal delivery $78.7 \%$ and $76.7 \%$, respectively, while $21.3 \%$ of cases and $23.3 \%$ of controls had caesarean section (C.S.) delivery, which indicates the absence of the significant differences between PPD and the type of delivery $(P=0.782)$. Despite that the mothers in the control group were breast-feeding $60.0 \%$ compared to the cases group $50.7 \%$, the results in this study, no statistical association between PPD and the lactation $(P=0 \cdot 212)$. The results showed statistically significant associations between PPD and being classified as a high-risk pregnancy $(P=0.003)$, in which $6.0 \%$ of the cases mothers faced gestational hypertension, $3.3 \%$ faced gestational diabetes and $2.0 \%$ had pre-term labour. Previous use of oral or injection contraceptive methods did not indicate any significant differences between the two groups $(P=0 \cdot 178)$.

Table 1 also shows that the mothers who had an undesired and uncomfortable feeling during the last pregnancy period were more susceptible to develop PPD after the baby delivery, as for $58.0 \%$ of the mothers in the cases group were suffering from this such issues, and thus there were statistically significant differences between the two 


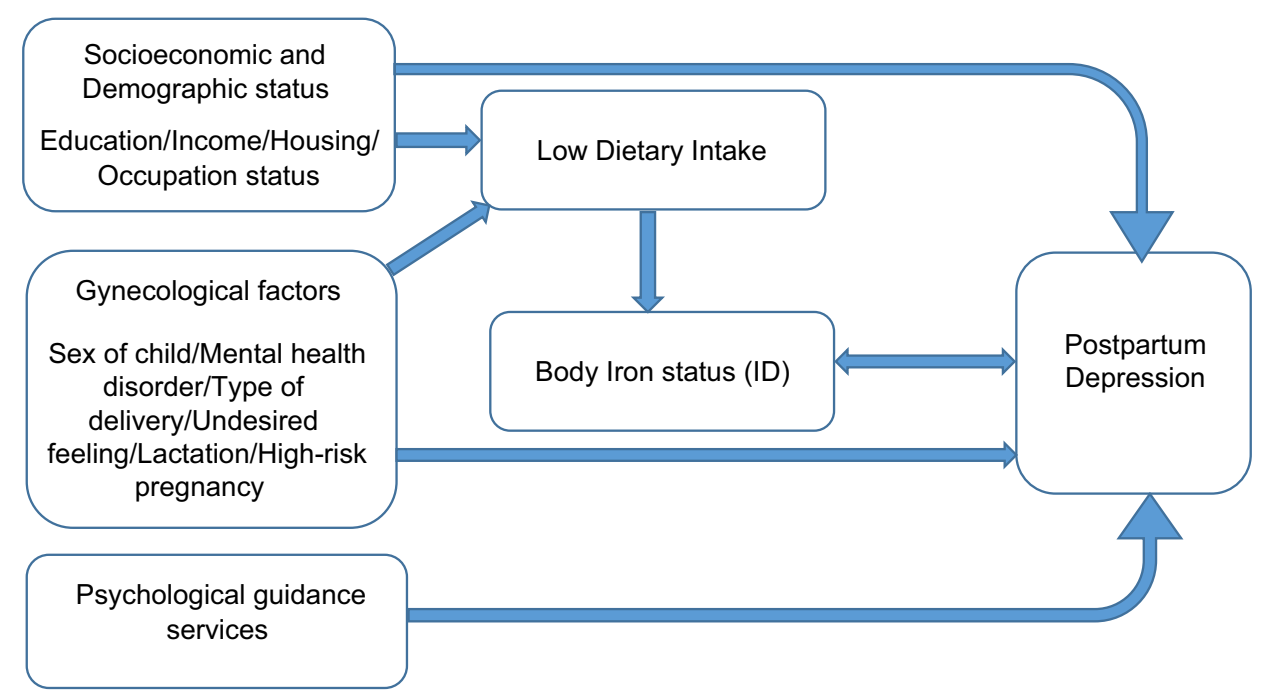

Fig. 1 (colour online) Conceptual frame of the study

Table 1 Comparison between cases and control

\begin{tabular}{|c|c|c|c|}
\hline Profile & Cases (PPD) $(\%)=150$ & Controls (non-PPD) $(\%)=150$ & $P$-value \\
\hline \multicolumn{4}{|l|}{ Housing } \\
\hline Ownership & 88.0 & $89 \cdot 3$ & \multirow[t]{2}{*}{0.856} \\
\hline Rent & $12 \cdot 0$ & 10.7 & \\
\hline \multicolumn{4}{|l|}{ Educational level of the mother } \\
\hline Less than high school & $10 \cdot 0$ & $9 \cdot 3$ & \multirow[t]{4}{*}{1.00} \\
\hline \multirow{4}{*}{\multicolumn{4}{|c|}{$\begin{array}{l}\text { school } \\
\text { High school } \\
\text { University and equivalent }\end{array}$}} \\
\hline & & & \\
\hline & & & \\
\hline & & & \\
\hline Employed & 3.3 & 6.7 & \multirow[t]{2}{*}{0.289} \\
\hline House wife & $96 \cdot 7$ & $93 \cdot 3$ & \\
\hline \multicolumn{4}{|l|}{ Income category } \\
\hline Deep poverty & $95 \cdot 3$ & $92 \cdot 0$ & \multirow[t]{3}{*}{0.388} \\
\hline Poor & 3.3 & $4 \cdot 0$ & \\
\hline Not poor & 1.3 & $4 \cdot 0$ & \\
\hline \multicolumn{4}{|l|}{ Sex of child } \\
\hline Male & $56 \cdot 7$ & $50 \cdot 0$ & \multirow[t]{2}{*}{0.298} \\
\hline Female & $43 \cdot 3$ & $50 \cdot 0$ & \\
\hline Worried about the sex of the baby & $45 \cdot 3$ & $46 \cdot 0$ & 1.000 \\
\hline First child & $32 \cdot 0$ & $25 \cdot 3$ & 0.250 \\
\hline Planned pregnancy & $32 \cdot 7$ & 37.3 & 0.468 \\
\hline \multicolumn{4}{|l|}{ Type of delivery } \\
\hline Normal & $78 \cdot 7$ & $76 \cdot 7$ & \multirow[t]{2}{*}{0.782} \\
\hline C.S. & $21 \cdot 3$ & $23 \cdot 3$ & \\
\hline \multicolumn{4}{|l|}{ Lactation } \\
\hline Breast milk & $50 \cdot 7$ & $60 \cdot 0$ & \multirow[t]{3}{*}{0.212} \\
\hline Formula feeding & $4 \cdot 0$ & $2 \cdot 0$ & \\
\hline Mixed & $45 \cdot 3$ & 38.0 & \\
\hline High-risk pregnancy (last pregnancy) & $12 \cdot 0$ & $2 \cdot 7$ & 0.003 \\
\hline Contraceptive methods (oral or injection) & $16 \cdot 7$ & $10 \cdot 7$ & 0.178 \\
\hline Undesired feeling in the last pregnancy & $58 \cdot 0$ & $42 \cdot 0$ & 0.008 \\
\hline Taken psychological guidance services & $2 \cdot 0$ & $20 \cdot 7$ & $<0.001$ \\
\hline Mental health disorder in the last pregnancy & 58.0 & $29 \cdot 3$ & $<0.001$ \\
\hline
\end{tabular}

groups $(P=0.008)$. The relationship between the PPD and attending psychological guidance services was highly statistically significant $(P<0 \cdot 001)$, since $20.7 \%$ of the controls attended such services, while $98.0 \%$ of the cases did not attend any psychological guidance services before or during the pregnancy. Moreover, there was a highly significant association between the PPD and having one of the mental health disorder in the last pregnancy $(P<0 \cdot 001), 12.7 \%$ of the mothers in the cases group were suffering from major depression during the last pregnancy, $22.0 \%$ were suffering from eating disorders and $23.3 \%$ were suffering from either anxiety, insomnia or panic. 
Table 2 Haematological and biochemical characteristics of cases and controls

\begin{tabular}{|c|c|c|c|c|c|c|}
\hline \multirow[b]{2}{*}{ Profile } & \multicolumn{2}{|c|}{ Cases (PPD) $n 150$} & \multicolumn{2}{|c|}{$\begin{array}{c}\text { Controls (non-PPD) } \\
n 150 \\
\end{array}$} & \multirow[b]{2}{*}{$95 \% \mathrm{Cl}$} & \multirow[b]{2}{*}{$P$-value } \\
\hline & $\% /$ Mean & SD & (\%)/Mean & SD & & \\
\hline $\mathrm{Hb}(\mathrm{g} / \mathrm{dl})$ & 11.47 & 1.22 & $12 \cdot 08$ & 1.03 & $-0.86,-0.35$ & $<0.001$ \\
\hline $\operatorname{HCT}(\%)$ & 34.51 & $3 \cdot 14$ & $36 \cdot 07$ & $2 \cdot 79$ & $-2.23,-0.88$ & $<0.001$ \\
\hline MCV (fl) & $79 \cdot 23$ & $7 \cdot 40$ & $81 \cdot 29$ & 6.94 & $-3.69,-0.43$ & 0.013 \\
\hline Ferritin & 33.4 & $34 \cdot 81$ & $46 \cdot 06$ & 36.02 & $-20 \cdot 71,-4.62$ & $<0.001^{*}$ \\
\hline Low & $43 \cdot 30$ & & $15 \cdot 30$ & & & $<0.001$ \\
\hline Normal & $56 \cdot 70$ & & 84.7 & & & \\
\hline hs-CRP & $6 \cdot 22$ & $14 \cdot 75$ & $5 \cdot 23$ & $5 \cdot 74$ & $-1.55,3.53$ & $0.730^{*}$ \\
\hline Normal & $69 \cdot 30$ & & $69 \cdot 30$ & & & 1.00 \\
\hline High & $30 \cdot 7$ & & $30 \cdot 7$ & & & \\
\hline STfR & $32 \cdot 43$ & 35.90 & $20 \cdot 6$ & $23 \cdot 15$ & $4.96,18.69$ & $<0.001^{*}$ \\
\hline Low & 4.7 & & $10 \cdot 0$ & & & $<0.001$ \\
\hline Normal & $60 \cdot 0$ & & $75 \cdot 3$ & & & \\
\hline High & $35 \cdot 3$ & & $14 \cdot 7$ & & & \\
\hline sTfR/log ferritin index & $7 \cdot 29$ & 6.44 & $10 \cdot 46$ & 4.99 & $0356,1.34$ & $<0.001$ \\
\hline ID & $15 \cdot 3$ & & $4 \cdot 7$ & & & 0.003 \\
\hline Non-ID & 84.7 & & $95 \cdot 3$ & & & \\
\hline
\end{tabular}

HCT, haematocrit; MCV, mean corpuscular volume; sTfR, soluble transferrin receptors; ID, iron deficiency.

*Mann-Whitney test for non-parametric test.

Table 2 presents a highly significant differences between the two groups regarding the following $\mathrm{CBC}$ parameters that have a role in screening and diagnosis of the body iron status namely, $\mathrm{Hb}(P<0.001)$, haematocrit $(P<0.001)$ and mean corpuscular volume $(P=0.013)$. The table shows that most of the participants were not having inflammation since $69.3 \%$ of them had a normal hs-CRP level $(<5 \mathrm{mg} / \mathrm{l})$ and the rest of the participants had high hsCRP level (>5 mg/l).

The mean difference in the sTfR between the two groups was statistically significant and as shown in the table. The results show that cases have almost 12 units of sTfR higher than that of the controls. Moreover, the mean difference in the sTfR/log ferritin index was statistically significant and it was in favour of the control group. The sTfR/ $\log$ ferritin index determined that most $(84.7 \%)$ of the mothers in the cases group were not categorised with ID and 23 mothers $(15 \cdot 3 \%)$ were categorised with ID, while only 7 ( $4.7 \%)$ mothers in the controls group were categorised with ID, and $95.3 \%$ were not categorised with ID. Thus, there was a statistical significant differences in ID between the two groups $(P=0.003)$.

Based on the results of simple logistic regression (SLgR), independent variables with a $P$-value $<0.25$ or any other important associated factors from previous studies were included in the multiple logistic regression (MLgR). Table 3 presents all the determinants appearing in the final model that remained significantly associated with the PPD status, namely, the psychological guidance services, the undesired feeling during the last pregnancy, the mental health disorder in the last pregnancy and the body iron status. The results of MLgR showed that the mothers who did not attend any psychological guidance services were 8.54 times more likely to develop PPD (OR ${ }^{\text {adj }} 8 \cdot 54$, (95\% CI 2.41, 30.21); $P=0.001)$, and that the mothers who had undesired feeling in the last pregnancy were 1.77 times more susceptible to suffer from PPD than the mothers who did not have that feeling (OR ${ }^{\text {adj }} 1.77$, (95\% CI 1.04, 3.03); $P=0.034$ ).

The findings of the MLgR reveal that there was a statistically significant association between PPD and mental health disorders in the last pregnancy $(P=0 \cdot 001)$, as the mothers who suffered from major depression or eating disorders were three times more likely to be PPD (OR ${ }^{\text {adj }} 3 \cdot 56$, (95\% CI 1.36, 9.34); $P=0.010)$ and (OR ${ }^{\text {adj }} 3 \cdot 33$, (95\% CI $1.57,7.06) ; P=0.002$ ), respectively, while mothers who faced anxiety, insomnia or panic disorder were two times more likely to develop PPD than the mothers who did not suffer from mental health disorders (OR ${ }^{\text {adj }} 2 \cdot 54$, (95\% CI, $1.3,4.99) ; P=0.007)$. In investigating the association between PPD and body iron status, findings of this study imply that a significant association between PPD and body iron status existed $(p=0.015)$. The mothers who suffered from ID were three times more likely to have PPD (OR ${ }^{\text {adj }}$ 3.25, (95\% CI 1.26, 8.40); $P=0 \cdot 015)$.

\section{Discussion}

The recurrent wars on Gaza exacerbated the humanitarian crisis, unemployed in Palestine reached $31 \%$; of which $52 \%$ in Gaza while the poverty percentage among Palestinian individuals according to consumption patterns was $29 \%$, of which $53 \%$ of which was in Gaza, and almost three in four people are food-insecure ${ }^{(27,28)}$. As mentioned earlier, the degraded socio-economic situation and foodinsecure and the political violence makes depression one of the top five causes of disability in the occupied Palestinian territory. The intended aims of the present study were to investigate the role of body iron status role in PPD, in addition to other factors. 
Table 3 Factors associated with postpartum depression from multiple logistic regression analysis ( $n$ 300)

\begin{tabular}{|c|c|c|c|c|c|c|c|c|}
\hline \multirow[b]{2}{*}{ Variables } & \multicolumn{3}{|c|}{ Simple logistic regression } & \multicolumn{5}{|c|}{ Multiple logistic regression } \\
\hline & Odd ratio & $95 \% \mathrm{Cl}$ & $P$-value & Adjusted $\beta$ & SE & Adjusted OR & $95 \% \mathrm{Cl}$ & $P$-value \\
\hline Absence of psychological guidance services & $12 \cdot 76$ & $3 \cdot 81,42 \cdot 78$ & $<0.001$ & $2 \cdot 24$ & 0.64 & 8.54 & $2 \cdot 41,30 \cdot 21$ & 0.001 \\
\hline Undesired feeling in the last pregnancy & 1.91 & $1.21,3.02$ & 0.006 & 0.57 & 0.27 & 1.77 & $1.04,3.03$ & 0.034 \\
\hline Mental health disorder in the last pregnancy & - & & $<0.001$ & - & & - & & 0.001 \\
\hline Major depression & 4.57 & $1.82,11.47$ & 0.001 & 1.27 & 0.49 & 3.56 & $1 \cdot 36,9 \cdot 34$ & 0.010 \\
\hline Eating disorders & 3.97 & $1.97,7.97$ & $<0.001$ & $1 \cdot 20$ & 0.38 & 3.33 & $1.57,7.06$ & 0.002 \\
\hline Anxiety, insomnia or panic disorder & 2.56 & $1.39,4.72$ & 0.003 & 0.93 & 0.34 & 2.54 & $1.3,4.99$ & 0.007 \\
\hline Body iron status & 3.7 & $1.54,8.91$ & 0.004 & $1 \cdot 18$ & 0.48 & $3 \cdot 25$ & $1.26,8.40$ & 0.015 \\
\hline
\end{tabular}

OR ${ }^{\text {adj: }}$ OR adjusted.

The results of a prospective observational study by Chandrasekaran and his team showed that there was no difference in $\mathrm{Hb}$ or iron levels in women who had PPD compared to those without ${ }^{(17)}$. This contradicts the results of our study, since the results showed a highly significant association between PPD and $\mathrm{Hb}$ and haematocrit. In addition to a significant association with mean corpuscular volume $(P=0 \cdot 013)$, which could be due to the small sample size in Chandrasekaran et al. study that included only 103 mothers. On the other hand, there were many studies that correspond to the results of our study regarding the significant association between PPD and $\mathrm{Hb}$, as in the multivariate analysis of a study by Beard et al. which showed that the mother's scores on the EPDS were significantly correlated with $\mathrm{Hb}$ and mean corpuscular volume ${ }^{(29)}$. In addition, another study that involved 352 Saudi postpartum mothers depends only on Hb testing, as its low level detect of anaemia among the delivered mothers. The results of the study suggested that early postpartum anaemia evident by low $\mathrm{Hb}$ level is a significant risk factor for $\mathrm{PPD}^{(30)}$.

In the current study, the ferritin level in mothers with PPD was lower than its level in mothers without PPD. Also, the results of this study showed a highly significant association between PPD and serum ferritin level of the postpartum mothers. The same significant differences were detected between mothers with PPD and mothers without PPD regarding sTfR and body iron status (sTfR/log ferritin index). The results of the study did not detect any significant association between PPD and the hs-CRP level $(P=1.00)$. This significant relationship can be explained by the biological role of iron in maintaining the brain and cognition health since iron is critical for adequate myelination, neurotransmitter metabolism and function, and neuronal cellular and oxidative processes ${ }^{(17)}$. Thus low iron level will lead to a deterioration of these functions and develop the clinical depression in mothers. Likewise, there were studies in which results are consistent with the results of our study as the Albacara et al. study that supports the role of iron in the aetiology of PPD. The study observed a strong association between ferritin and $\operatorname{PPD}^{(14)}$, while there was no significant association between CRP and PPD $(P=0.51)$. The association between PPD and iron was also detected in a randomised double-blind placebo-controlled trial by Sheikh et al. which concluded that providing early iron supplementation to the delivered mothers, who suffer from PPD, can significantly improve the iron stores and lead to significant improvement in PPD with a $42.8 \%$ improvement rate during 6 weeks and that the persistence of PPD may be relevant to the lower postnatal ferritin levels in untreated mothers ${ }^{(15)}$. In contrast, results of study by Armony-Sivan et al. who investigated this association among Chinese mothers demonstrated that even in this large sample, no correlation reached statistical significance, as the correlations between the mothers iron biochemical tests $(\mathrm{Hb}$, mean corpuscular volume, ferritin, sTfR and sTfR Index) during mid- or late-pregnancy or 3 d postpartum. EPDS scores shortly after delivery or at 6 weeks were also low. Besides that, EPDS scores in anaemic and non-anaemic mothers did not differ from the results of Armony-Sivan et al. study, which showed similarity in iron status in both groups (women with or without PPD) ${ }^{(18)}$. Thus, there was no relationship between the maternal iron status and PPD. Moreover, there was an exception for sTfR at mid- and late-pregnancy and EPDS at 6 weeks, as higher sTfR was related to lower depressive symptoms, which was opposite from what would be expected.

The current study found that the mothers who suffered from ID were three times more likely to have PPD. However, the discrepancy between our results and the results of the other studies regarding the biochemical part could be due to the differences in the methodologies of performing the test including the device and the kits that been used to assess the biochemical markers since some normal ranges could differ from kit to another. Furthermore, we could not find any study that depends on the sTfR/log ferritin index to assess body iron status and its association with PPD.

The study detected a highly significant association between PPD and having one of the mental health disorders in the last pregnancy including major depression, eating disorders, anxiety, insomnia or panic. Our results are consistent with previous studies, as in the retrospective controlled study by Morgan et al., which showed that, during pregnancy, active bulimia (one of the eating disorders) is associated with postnatal depression, miscarriage and pre-term delivery. In addition, bulimia may be a treatable 
cause of the adverse obstetric outcome ${ }^{(31)}$. While in Stewart work that investigated the depression and anxiety showed that most PPD and anxiety were preceded by antenatal depression and anxiety. Furthermore, the antenatal anxiety also predicted PPD, even after controlling for antenatal depression $^{(32)}$.

On the other hand, the results of our study showed a significant difference between PPD mothers and non-PPD mothers regarding the classification of the mother as high-risk pregnancy during the last pregnancy. This corresponds to the results of a study by Zadeh $e t$ al. which found that the prevalence of moderate-to-severe symptoms of depression and anxiety was higher among mothers who had a high-risk pregnancy ${ }^{(33)}$.

Overall, there was a discrepancy between the results of this study and the results of the numerous previous studies that investigated those factors. This could be due to many reasons as the variation in studies environment, habits, concepts and beliefs, in addition to the diversity regarding the methodology that has been used. Furthermore, the variation between the studies in selecting the cut-off point in the EPDS could be one of the most important reasons.

The results of the current study indicate that undesired feeling in the last pregnancy, mental health disorder and high-risk pregnancy are some of the PPD factors. These factors combined together can also worsen the condition of PPD in the affected mothers, as these factors are usually combined with affecting mother's appetite for food. This will lead to a deficiency of some basic elements such as ID, which in turn, will aggravate the situation.

\section{Strengths}

Choosing the mothers after a month of postpartum helped prevent overlapping with other postpartum psychiatric disorders including the postpartum blues and psychosis. Furthermore, the translated copy of the EPDS questionnaire including the used cut-off point (scoring of 10 and higher) was valid and pre-tested, whereas the framing of the questionnaire questions and their answers in addition to the selection of the cut-off point are considered critical in detecting the presence of PPD among the mothers.

Regarding the detection of ID, the effect of inflammation (high CRP concentration) on the ferritin was avoided, by the use of STfR that reflects the functional iron compartment, and is not acute phase reactant. Moreover, the equation that was used (sTfR/log ferritin index) in detecting the body iron stores has been shown to be valid and superior to routine tests.

\section{Limitations}

Despite all the precautions that were taken to make the participants feel safe and comfortable while answering the questions, it is believed that some of them were embarrassed to answer some questions correctly.
Using sTfR/log ferritin index as a proxy for iron status can be considered an addition point, but on the other hand, it can be considered a negative point, as it deprived the researchers of comparisons with other studies.

Case-control studies are an efficient method for the study of rare outcomes but suffer various limitations, including susceptibility to bias in recollection about exposure, and reverse causality

Due to the financial barriers, the biochemical tests were restricted to the selected tests. Other complementary tests could not be performed, as well as the anthropometric measurements could not be performed due to minimising interview times out of consideration for the circumstances of the participating mothers.

\section{Conclusion}

The results showed that mothers who have ID are at high risk of developing PPD, as the mothers who suffered from ID were three times more likely to have PPD.

\section{Recommendations}

The mothers and their families need to pay more attention to the mother's food consumption before, during and after pregnancy. Primarily, ensure diversity in her consumed food and focus on foods rich in iron so she can meet her recommended daily intake. Furthermore, the mother must be aware of her feelings and psychological state and should not hesitate to attend the available psychological guidance services before, during or after the postpartum period. Routine physical and psychological check-ups during pregnancy period are highly recommended particularly for mental health disorders.

\section{Acknowledgements}

Acknowledgements: We thank the mothers who gave us their time, the Palestinian Ministry of Health for guaranteed access to Primary Health Care centres. Our gratitude is extended to Al-Azhar University-Gaza academic staff for their continued support and cooperation. Our sincere thanks to ECNAD team for their guidance assistance in accomplishing this work. This is to indicate that this study has not received any funds from any party. Also our thanks extended to Jennifer Kastner for or her effort in English language editing. Financial support: The authors have not received any financial support from anybody. Conflict of interest: There are no conflicts of interest. Authorship: S.A.H. designed and implemented the research, conducted data analysis and wrote the paper. I.A.N. and M.A.G were involved in designing and refining the study protocol and M.S.L. did the data analysis and reviewing the article. Ethics of buman subject participation: The present study was 
approved by the Helsinki Committee (PHRC/HC/633/19), the Deanship of the Faculty of Pharmacy at Al-Azhar University-Gaza and the Palestinian ministry of Health. Written consent was obtained from the parents of children and they were informed about their rights to reject or to withdraw from the study at any time.

\section{References}

1. WHO (2020) WHO Guidance Helps Detect Iron Deficiency and Protect Brain Development. https://www.who.int/ news-room/detail/20-04-2020-who-guidance-helps-detectiron-deficiency-and-protect-brain-development (accessed May 2020).

2. McArdle HJ, Gambling L \& Kennedy C (2014) Iron deficiency during pregnancy: the consequences for placental function and fetal outcome. Proc Nutr Soc 73, 9-15.

3. Gupta C (2014) Role of iron (Fe) in body. IOSR JAC 7, 38-46.

4. Vahdat Shariatpanaahi M, Vahdat Shariatpanaahi Z, Moshtaaghi M et al. (2006) The relationship between depression and serum ferritin level. Eur J Clin Nutr 61, 532-535.

5. Bozoky I \& Corwin EJ (2002) Fatigue as a predictor of postpartum depression. J Obstet Gynecol Neonatal Nurs 31, 436-443.

6. Turkoski B (2003) Tired blood: part 1. Orthop Nurs 22, 222-227.

7. Ashraf TS, De Sanctis V, Yassin M et al. (2017) Chronic anemia and thyroid function. Acta Biomed 88, 119-127.

8. Vigod SN, Villegas L, Dennis CL et al. (2010) Prevalence and risk factors for postpartum depression among women with preterm and low-birth-weight infants. Int J Gynecol Obstet 117, 540-550.

9. Pearlstein T, Howard M, Salisbury A et al. (2009) Postpartum depression. Am J Obstet 200, 357-364.

10. Yan J, Liu Y, Cao L et al. (2017) Association between duration of folic acid supplementation during pregnancy and risk of postpartum depression. Nutrients 9, 1206.

11. Huang Q, Liu H, Suzuki K et al. (2019) Linking what we eat to our mood: a review of diet, dietary antioxidants, and depression. Antioxidants 8, 376.

12. Hogg-Kollars S, Mortimore D \& Snow S (2011) Nutrition health issues in self-reported postpartum depression. Gastroenterol Hepatol Bed Bench 4, 120-136.

13. Brown JE, Isaacs J, Krinke B et al. (2010) Nutrition through the Life Cycle, 4th ed. Belmont: Cengage Learning.

14. Albacar G, Sans T, Martín-Santos R et al. (2011) An association between plasma ferritin concentrations measured $48 \mathrm{~h}$ after delivery and postpartum depression. J Affect Disord 131, 136-142.

15. Sheikh M, Hantoushzadeh S, Shariat M et al. (2017) The efficacy of early iron supplementation on postpartum depression, a randomized double-blind placebo-controlled trial. Eur J Nutr 56, 901-908.

16. Wassef A, Nguyen Q, St-André M et al. (2019) Anaemia and depletion of iron stores as risk factors for postpartum depression: a literature review. J Psychosom Obstet 40, 19-28.

17. Chandrasekaran N, De Souza LR, Urquia ML et al. (2018) Is anemia an independent risk factor for postpartum depression in women who have a cesarean section? A prospective observational study. BMC Pregnancy Childbirth 18, 400.

18. Armony-Sivan R, Shao J, Li M et al. (2012) No relationship between maternal iron status and postpartum depression in two samples in China. J Pregnancy 2012, 521431.

19. Jalambo M, Karim N, Naser I et al. (2018) Prevalence and risk factor analysis of iron deficiency and iron-deficiency anaemia among female adolescents in the Gaza Strip, Palestine. Public Health Nutr 21, 2793-2802.

20. Canetti D, Hall B, Greene T et al. (2014) Improving mental health is key to reduce violence in Israel and Gaza. Lancet 384, 493-494.

21. Department of Health \& Government of Western Australia (2006) Edinburgh Postnatal Depression Scale (EPDS): Translated Versions - Validated. Perth, Western Australia: State Perinatal Mental Health Reference Group; available at https://www.dchealthcheck.net/documents/10-2015-EPDSTranslations.pdf (accessed May 2020).

22. WHO (2004) Assessing the Iron Status of Populations: Report of a Joint World Health Organization/Centers for Disease Control and Prevention Technical Consultation on the Assessment of Iron Status at the Population Level. Geneva, Switzerland: World Health Organization.

23. Cogswell ME, Looker AC, Pfeiffer CM et al. (2009) Assessment of iron deficiency in US preschool children and non-pregnant females of childbearing age: national health and nutrition examination survey 2003-2006. Am J Clin Nutr 89, 1334-1342.

24. WHO (2014) Serum Transferrin Receptor Levels for the Assessment of Iron Status and Iron Deficiency in Populations. Geneva: World Health Organization.

25. Infusino I, Braga F, Dolci A et al. (2012) Soluble transferrin receptor (sTfR) and sTfR/log ferritin index for the diagnosis of iron-deficiency anemia a meta-analysis. Am J Clin Pathol 138, 642-649.

26. López M, Molinos F, Carmona M et al. (2006) Serum transferrin receptor in children: usefulness for determinating the nature of anemia in infection. JPHO 28, 809-815.

27. PCBS (2019) PCBS: On the Occasion of the International Population Day. Palestine: Palestinian Central Bureau of Statistics; available at http://www.pcbs.gov.ps/post.aspx? lang=en\&ItemID=3503 (accessed May 2020).

28. WFP (2019) Palestine Annual Country Report 2019 Country Strategic Plan 2018-2022. Palestine: World Food Programme; available at https://docs.wfp.org/api/docu ments/WFP-0000113833/download/ (accessed May 2020).

29. Beard JL, Hendricks MK, Perez EM et al. (2005) Maternal iron deficiency anemia affects postpartum emotions and cognition. J Nutr 135, 267-272.

30. Alharbi AA \& Abdulghani HM (2014) Risk factors associated with postpartum depression in the Saudi population. Neuropsychiatr Dis Treat 10, 311-316.

31. Morgan JF, Lacey JH \& Chung E (2006) Risk of postnatal depression, miscarriage, and preterm birth in bulimia nervosa: retrospective controlled study. Psychosom Med 68, 487-492.

32. Stewart D (2005) Depression during pregnancy. Can Fam Physician 51, 1061-1063.

33. Zadeh MA, Khajehei M, Sharif F et al. (2012) High-risk pregnancy: effects on postpartum depression and anxiety. $\mathrm{Br} \mathrm{J}$ Midwifery 20, 104-113. 